\title{
Polyhydroxyalkanoates: Waste glycerol upgrade into electrospun fibrous scaffolds for stem cells culture
}

\author{
Raphaël F. Canadas ${ }^{a, 1}$, João M.B.T. Cavalheiro ${ }^{a}$, João D.T. Guerreiro a \\ M. Catarina M.D. de Almeida ${ }^{\mathrm{a}, \mathrm{b}}$, Eric Pollet ${ }^{\mathrm{c}}$, Cláudia Lobato da Silva ${ }^{\mathrm{a}}$, \\ M.M.R. da Fonseca ${ }^{a}$, Frederico Castelo Ferreira ${ }^{a, *}$
}

\author{
a Department of Bioengineering and IBB - Institute for Biotechnology and Bioengineering, Instituto Superior Técnico, Universidade de Lisboa, Av. Rovisco \\ Pais, 1049-001 Lisboa, Portugal \\ ${ }^{\mathrm{b}}$ CiiEM - Center for Interdisciplinary Research Egas Moniz, Campus Universitário, Quinta da Granja, Monte de Caparica 2829-511, Caparica, Portugal \\ ${ }^{\mathrm{c}}$ LIPHT - Laboratoire d'Ingénierie des Polymères pour les Hautes Technologies, Ecole de Chimie, Polymères et Matériaux, Université de Strasbourg, 25 rue \\ Becquerel, 67087 Strasbourg Cedex 2, France
}

\section{A R T I C L E I N F O}

\section{Article history:}

Received 12 February 2014

Received in revised form 11 April 2014

Accepted 2 May 2014

Available online $\mathrm{xxx}$

\section{Keywords:}

Polyhydroxyalkanoates

Waste glycerol

Stem cell scaffolds

Electrospinning

Mesenchymal stem cell

\begin{abstract}
A B S T R A C T
This integrated study shows that waste glycerol can be bio-valorized by the fabrication of electrospun scaffolds for stem cells. Human mesenchymal stem cells (hMSC) provide an interesting model of regenerating cells because of their ability to differentiate into osteo-, chrondro-, adipo- and myogenic lineages. Moreover, hMSC have modulatory properties with potential on treatment of immunologic diseases. Electrospun fiber meshes offer tunable mechanical and physical properties that can mimic the structure of the native extracellular matrix, the natural environment where cells inhabit. Following a biorefinery approach, crude glycerol directly recovered from a biodiesel post-reaction stream was fed as major C source to Cupriavidus necator DSM 545 to produce polyhydroxyalkanoates at polymer titers of 9-25 g/L. Two of the $\mathrm{P}(3 \mathrm{HB}-4 \mathrm{HB}-3 \mathrm{HV})$ terpolymers produced, one containing $11.4 \% 4 \mathrm{HB}$ and $3.5 \% 3 \mathrm{HV}$ and the other containing $35.6 \% 4 \mathrm{HB}$ and $3.4 \% 3 \mathrm{HV}$, were electrospun into fibers of average diameters of 600 and $1400 \mathrm{~nm}$, respectively. hMSC were cultured for 7 days in both fiber meshes, showing their ability to support stem cell growth at acceptable proliferation levels. Comparative results clearly demonstrate that scaffold topology is critical, with electrospun PHA fibers succeeding on the support of significant cell adhesion and proliferation, where planar PHA films failed.
\end{abstract}

(c) 2014 Elsevier B.V. All rights reserved.

\section{Introduction}

Industrial efforts for microbial synthesis of polyhydroxyalkanoates (PHA) started with the pilot plant trial of Imperial Chemical Industries in 1980 with the aim of bringing environmental sustainability to the polymer sector, producing a biodegradable polymer to replace the petroleum based ones. PHAs are biological macromolecules that can be produced from different $\mathrm{C}$ sources besides

\footnotetext{
* Corresponding author at: Principal Research Scientist, IBB/DBE-Instituto Superior Técnico, Torre Sul, Av. Rovisco Pais, 1049-001 Lisboa, Portugal.

Tel.: +35121841 9598/91 68288 17; fax: +351218419062.

E-mail addresses: frederico.ferreira@ist.utl.pt, frederico_castelo@yahoo.com (F.C. Ferreira).

1 Present address: 3B's Research Group - Biomaterials, Biodegradable and Biomimetic, University of Minho, Headquarters of the European Institute of Excellence on Tissue Engineering and Regenerative Medicine, Avepark 4806-909 Taipas, Guimarães, Portugal.
}

refined sugars, namely sugar cane syrup molasses and vegetable oils [1]. Since 2003, as biodiesel production has become more prominent in the energy sector, the volumes of available glycerol in the European and US market increased, while the respective spot price decreased [2]. From 1995 to 2007, the price of refined glycerol dropped from US\$1 to US\$0.34 per pound and, in 2006, crude glycerol by-product of biodiesel was US $\$ 0.02$ per pound [3], calling for new uses of this commodity. As far as bioprocess robustness and cost efficiency are concerned, it is crucial to assess crude grade instead of highly purified glycerol as C source, since many microbial cultures fail due to traces of toxic compounds carried out from biodiesel production. Following a biorefinery approach, Cavalheiro et al. [4,5] have demonstrated the efficient use of crude glycerol as major C source for the biological synthesis of PHAs.

Synthetic polyesters such as polycaprolactone, polyglycolate and polylactate are polymers approved by regulatory agencies for medical applications [6,7]. Due to their ester moiety, these polymers are biodegradable into products tolerable by human 
metabolism at low concentrations and they have, therefore, been extensively tested as materials for the development of scaffolds for cell and tissue engineering, as reviewed elsewhere [8], two specific examples with clinical applications include cartilage regeneration [9] and engineered bladders [10]. Polyester hydrophobicity, elasticity, and biodegradability depend on the alkyl moiety, and block co-polymers, e.g. poly(lactic-co-glycolic acid) with different glycolide and lactide composition have been chemically synthesized to tailor such properties to biomedical requirements. Bio-produced PHAs are also polyesters composed by different monomers including the R-3-hydroxybutyrate (3HB), R-4-hydroxybutyrate (4HB), $\mathrm{R}-3$-hydroxyvalerate (3HV), R-3-hydroxyhexanoate (3HHx) and R-3-hydroxyoctanoate (3HO). According to their monomeric composition, polymers with different hydrophobicity and elasticity are obtained. In general, the Young's modulus and tensile strength increase with the increase of the $3 \mathrm{HB}$ molar fraction in co-polymers such as $\mathrm{P}(3 \mathrm{HB}-3 \mathrm{HV})$ and $\mathrm{P}(3 \mathrm{HB}-4 \mathrm{HB})$, whereas elasticity decreases [11-13]. Contrarily to other biopolymers with biomedical uses, such as collagen and hyaluronate, PHAs are thermoplastic and thus suitable to be processed by a wide range of techniques usually applied to synthetic polymers [14].

In 2007, the FDA approved the clinical application of $\mathrm{P}(4 \mathrm{HB})$ ("TephaFLEX ${ }^{\circledR}$ Absorbable Suture" and "BioTREK ${ }^{\mathrm{TM}}$ Bioabsorbable Septal Repair"). Since then, there has been an increasing interest in testing PHAs for the manufacture of scaffolds for cell and tissue engineering applications. Homopolymers $(\mathrm{P}(3 \mathrm{HB})$ and $\mathrm{P}(4 \mathrm{HB}))$, as well as co-polymers ( $\mathrm{P}(3 \mathrm{HB}-4 \mathrm{HB}), \mathrm{P}(3 \mathrm{HB}-3 \mathrm{HV})$ and $\mathrm{P}(3 \mathrm{HB}-3 \mathrm{HHx})$ ) and blends of different PHA types have been used for the fabrication of scaffolds with different properties [14-19]. An alternative strategy has been the use of PHAs combined with other materials such as polyvinyl alcohol, polylactatic acid [20] or chitosan [21]. Terpolymers had been successfully used as planar surfaces for cell cultivation [22,23], but not processed in 3D structures. Recent studies to provide 3D structures using PHAs include the fabrication of microspheres by solvent-water and solvent evaporation based protocols [15]. Plus, techniques such as particulate leaching [24], salt leaching [16], freeze drying [17] and thermal induced solid-liquid phase separation [18] have been used for the fabrication of porous scaffolds with different pore sizes according to the protocol and polymer used. Flat sheets have also been prepared and rolled [25]; in addition, fibrous structures have been processed by extrusion [12] and more often, by electrospinning [19-21]. Different cell types have been cultured on 2D or 3D PHA-based scaffolds, including L929 mouse fibroblast cells [21], used for cytotoxicity tests, other animal and human cell lines [15,20,24], and stem cells [17-19]. In addition, different in vivo studies show good biocompatibility of these materials, their ability to support cell growth and their low level of inflammatory response. Examples with animal trials that show no adverse effects of PHAs are (i) a study where PHB and $\mathrm{P}(3 \mathrm{HB}-3 \mathrm{HV})$ were implanted as sutures to treat muscle facial cuts in Wistar rats [11] and (ii) PHB being used as an artificial nerve conduit with adipose-derived stem cells transplanted into rat sciatic nerve model, with the aim of releasing growth factors for stimulation of endogenous Schwann cells activity [14].

The mechanical properties and the biocompatibility of PHAs significantly vary with their chemical composition and the processing mode. Therefore, in the current study, the homopolymer $\mathrm{P}(3 \mathrm{HB})$ and three terpolymers directly produced from crude waste glycerol are firstly assessed for material bioprocessing cytotoxicity and biocompatibility using L929 mouse fibroblast. Then, PHAs ability to support tissue engineering strategies was assessed through seeding and cultivation of human bone marrow (BM) derived mesenchymal stem cells (hMSC) on the PHA scaffolds. MSC are anchorage dependent stem cells with a high potential for regenerative due to their potential to differentiate in osteo-, chrondro-, adipo- [26] and myogenic [27] lineages, as well as their immune modulatory properties, which can be explored, for example on treatment of graft-vs-host disease and auto-immune disorders [28]. Taking into account the small amount of available hMSC in vivo and the large numbers usually required for their therapeutic application, a stage of ex vivo cultivation of hMSC involving seeding, expansion and, in some cases, differentiation is often required. Then, cells can be harvested for administration or an ex vivo seeded scaffold can be implanted in vivo. In the latter case, focusing tissue engineering settings, the scaffold biodegradability rate is an important issue.

In this work, scaffolds were prepared by electrospinning of a polymer solution. This method allows the production of continuous fiber meshes with high specific surface areas and diameters ranging from 40 to $4000 \mathrm{~nm}$ [29]. Electrospinning has been widely used in the manufacture of non-woven fiber meshes for tissue engineering applications. Electrospun fibers meshes offer tunable mechanical and physical properties that can mimic the structure of the native extracellular matrix (ECM), the natural environment where cells reside. It is noteworthy that one of the main structural components of the ECM is collagen, with structure fiber bundle diameters ranging in the nanoscale [30]. Electrospun nanofibers of biocompatible polymers can provide a good synthetic scaffold for hMSC cultivation by mimicking the ECM in size and structure. Indeed, the BM niche where MSC are found is characterized by an interplay of soluble factors, cell-to-cell, and cell to ECM components interactions [31]. Previous studies using electrospun scaffolds for hMSC show the ability to improve cell adhesion and proliferation, as well as the effect of scaffold architecture in cell response [32,33] and differentiation toward endothelial lineages [19].

PHA properties (e.g. stiffness, hydrophobicity and biodegradability) can be tuned according to the structure of the biomacromolecules produced. Importantly, the current work uses crude glycerol as carbon source for the production of PHA terpolymers, which are then, for the first time, employed on the manufacturing of electrospun scaffolds for stem cells proliferation. The approach taken illustrates the potential to combine PHA bioproduction conditions to tailor polymer properties and electrospinning technique to provide a scaffold 3D structure that mimics the stem cell niche. The results are expected to contribute toward sustainability of both biodiesel and biodegradable plastics production (Fig. 1).

\section{Materials and methods}

\subsection{PHA production and recovery}

PHAs were bioproduced in a $2 \mathrm{~L}$ reactor (B. Braun M2 culture vessel coupled to a Biostat MD 884402/0 and a digital control unit $884201 / 9$ ) by two stage fed-batch cultivation of Cupriavidus necator DSM 545. Cell growth was performed under balanced conditions (growth stage) and PHA synthesis was subsequently promoted by nitrogen starvation. The glycerol-rich-phase (GRP) from a biodiesel plant (Fábrica Torrejana de Biocombustíveis, S.A., Portugal) was utilized as major $C$ source. Gamma-butyrolactone (GBL) and propionic acid (PA), both from ACROS, purity 99\%, were used as precursors for the $4 \mathrm{HB}$ and $3 \mathrm{HV}$ monomers, respectively. GBL and PA were fed to the system in amounts 10 and 100 fold lower, respectively, than those of biodiesel glycerol. Four batches were carried out and labeled from A to D. The specific conditions used for each batch concerning $C$ sources, dissolved oxygen concentrations (DOC) and cultivation time per stage are shown in Table 1. Cultivation protocols for the production of $\mathrm{P}(3 \mathrm{HB})$ and $\mathrm{P}(3 \mathrm{HB}-4 \mathrm{HB}-3 \mathrm{HV})$ have been previously described [4,5]. In the current study minor changes were introduced: (i) during the growth phase, the glycerol concentration was controlled within the range $20-60 \mathrm{~g} / \mathrm{L}$; (ii) in batch $\mathrm{B}$ the GBL pulse at the beginning of the nitrogen starvation stage was $6 \mathrm{~g} / \mathrm{L}$. Information on monomers quantification, strain storage, inoculum preparation, composition 


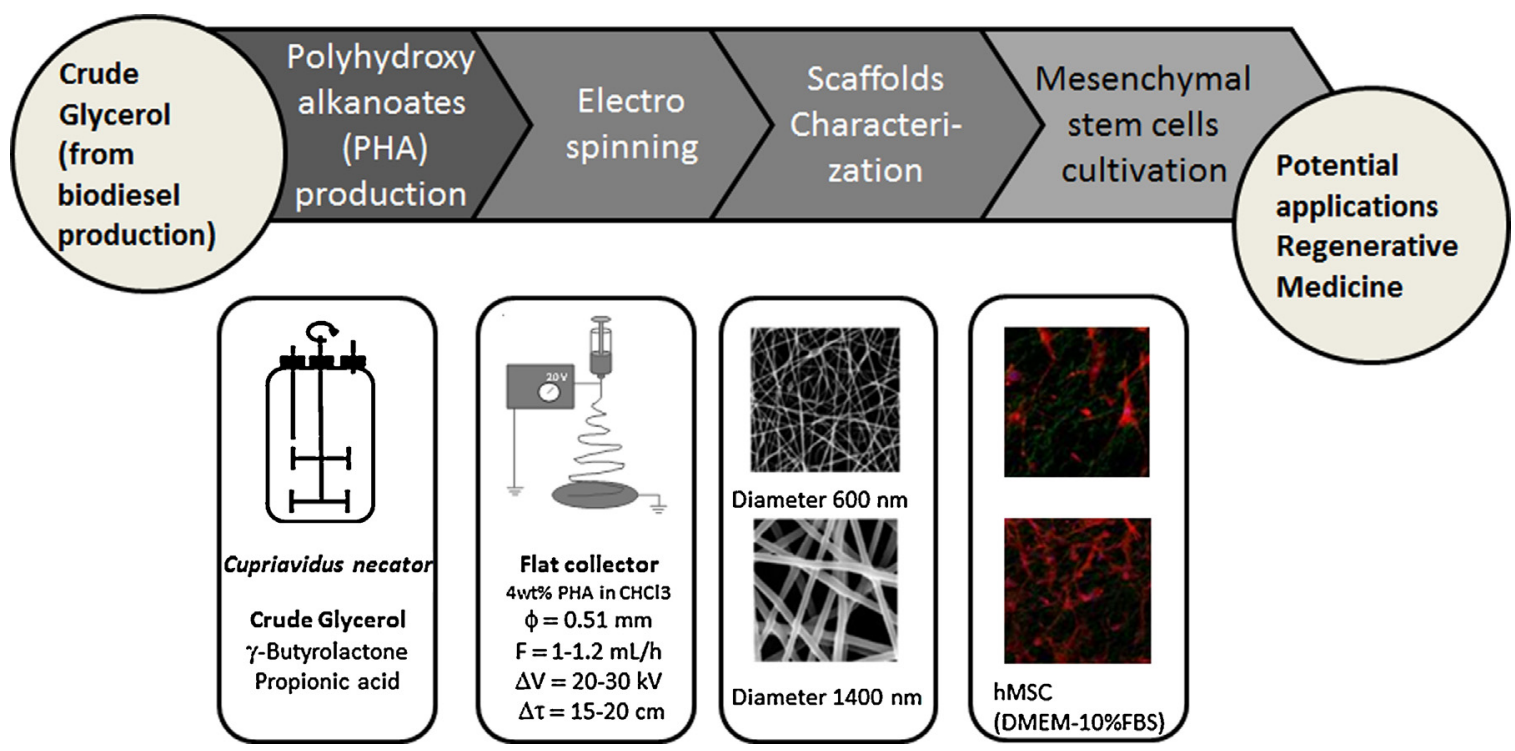

Fig. 1. Schematic of integrated approach for waste glycerol bio-valorization into fabrication of electrospun scaffolds for stem cells.

of inocula, cultivation media, waste glycerol characterization and PHA extraction from lyophilized cells is available elsewhere [4,5].

\subsection{PHA characterization and preparation of films}

PHA solutions of $10-15 \mathrm{mg} / \mathrm{mL}$ were prepared in chloroform and the PHA monomeric composition was determined by ${ }^{1} \mathrm{H}$ NMR (Bruker $400 \mathrm{MHz}$ ). Quantification of the $3 \mathrm{HV}, 3 \mathrm{HB}$ and $4 \mathrm{HB}$ content, as well as estimation of PHA molecular weight (MW) and polydispersity index (PI) by size exclusion chromatography is available elsewhere [34]. PHA films with average thickness between 100 and $450 \mathrm{~mm}$ were prepared from chloroform PHA solutions (ca. $60 \mathrm{mg} / \mathrm{mL}$ ) using a casting knife. Uniaxial tensile tests were performed using a MTS2/M universal testing machine, at $25^{\circ} \mathrm{C}$ and $10 \mathrm{~mm} / \mathrm{min}$ crosshead speed on rectangular tensile bars $(50 \mathrm{~mm}$ length $\times 10 \mathrm{~mm}$ width; $30 \mathrm{~mm}$ between grips). The average tensile strength at rupture (TSR), percentage of deformation at break $(\varepsilon)$ and Young's modulus $(E)$ were calculated from the stress-strain curves using TestWorks4 software. Water static contact angles were measured by the sessile drop method [35]. Drops of 2-3 $\mu \mathrm{L}$ generated with a micrometric syringe were deposited on the substrates surface. Images sequences recorded during 60 using a video camera (JAI CV-A50), a microscope (Wild M3Z) and a frame grabber (Data Translation model DT3155) were analyzed by Axisymmetric Drop Shape Analysis-Profile (ADSA-P) software. Results presented are the average of at least six experiments.

\subsection{PHA electrospinning and fiber processing}

PHA in chloroform solutions $(4 \%(\mathrm{w} / \mathrm{v})$ were electrospun through a spinneret with $0.51 \mathrm{~mm}$ inner diameter, connected by a teflon tube to a syringe pump (KDSLegato210) set at $1-1.2 \mathrm{~mL} / \mathrm{h}$, under applied voltage of 20-30 kV (Glassman, 1 ES/EL40P01) and fibers were collected on a flat stainless steel plate placed at $15-20 \mathrm{~cm}$ from the spinneret. Fibrous membranes obtained were placed under vacuum for $3 \mathrm{~h}$ for solvent traces removal, sterilized by UV light, washed (PBS, GibcoBRL) and incubated with medium.

\subsection{Cytotoxicity tests, mesenchymal stem cell culture and characterization}

Medium used for L929 mouse fibroblasts (L929-mF) and MSC cultivation and materials conditioning, if not mention otherwise, was Dulbecco's modified Eagle's medium (DMEM) supplemented with $10 \%(\mathrm{v} / \mathrm{v})$ of fetal bovine serum (FBS) and with streptomycin $(0.025 \mathrm{mg} / \mathrm{mL})$ and penicillin $(0.025 \mathrm{U} / \mathrm{mL})$, from GibcoBRL. Incubation conditions were $37{ }^{\circ} \mathrm{C}$ and $5 \% \mathrm{CO}_{2}$ in humidified atmosphere.

\subsubsection{Cytotoxicity tests}

PHA samples were tested for cytotoxicity following the ISO10993-5 guidelines for indirect and direct contact assays as previously described [36] (in quatriplicate). For indirect tests medium and materials were incubated for 3 days and medium

Table 1

Conditions used to obtain the various PHAs tested throughout this work in the cultivations of $C$. necator on GRP.

\begin{tabular}{|c|c|c|c|c|c|c|c|c|c|}
\hline \multirow[t]{3}{*}{ Batch } & \multicolumn{9}{|c|}{ Co-substrates (g/L) } \\
\hline & \multicolumn{2}{|l|}{ GBL } & \multicolumn{2}{|l|}{ PA } & \multicolumn{2}{|l|}{ DOC (\%) } & \multicolumn{3}{|c|}{ Cultivation time (h) } \\
\hline & $\begin{array}{l}\text { Growth } \\
\text { stage }\end{array}$ & $\begin{array}{l}\text { Nitrogen starvation } \\
\text { stage }\end{array}$ & $\begin{array}{l}\text { Growth } \\
\text { stage }\end{array}$ & $\begin{array}{l}\text { Nitrogen starvation } \\
\text { Stage }\end{array}$ & $\begin{array}{l}\text { Growth } \\
\text { stage }\end{array}$ & $\begin{array}{l}\text { Nitrogen starvation } \\
\text { stage }\end{array}$ & $\begin{array}{l}\text { Growth } \\
\text { stage }\end{array}$ & $\begin{array}{l}\text { Nitrogen } \\
\text { starvation }\end{array}$ & Total \\
\hline A & 0 & - & - & - & 20 & 2 & 26 & 19 & 45 \\
\hline B & 1.8 & $3-10$ & - & $0-2$ & 20 & 2 & 26 & 24 & 50 \\
\hline $\mathrm{C}$ & 1.8 & $10-20$ & - & $0-2$ & 20 & 2 & 22 & 23 & 45 \\
\hline $\mathrm{D}$ & 1.8 & $10-20$ & - & $0-2$ & 20 & 20 & 23 & 44 & 67 \\
\hline
\end{tabular}

The GRP concentration was kept at 20-60 g/L and 10-40 g/L during the growth and the nitrogen starvation stages, respectively. 
used to culture L929 cells (seeded at $80 \times 10^{3}$ cells $/ \mathrm{cm}^{2}$ ) on 24 -well plates (BD Falcon) for 3 days, the cell metabolic activity determined using the using a MTT Cell proliferation kit (Sigma-Aldrich, CGD-1). Fresh medium and fresh medium with $0.5 \mathrm{v} / \mathrm{v}$ ethanol $96 \%$ were used as negative and positive controls for cytotoxicity, respectively. In direct contact assays, $\mathbf{L} 929$ cells (seeded at 3000 cells $/ \mathrm{cm}^{2}$ ) in 6 -well plates (BD Falcon) for 3 days until confluence; then PHA samples were incubated for 3 days in the top of cell layer and formation of cell halo assessed by inverted fluorescence microscopy (Leica DMI3000B).

\subsection{2. hMSC isolation}

$\mathrm{BM}$ aspirates were harvested after informed donor consent and BM hMSC were isolated as described elsewhere [37] and stored in liquid nitrogen. Upon thawing, BM-hMSC were expanded for 3-5 passages in a tissue flask. Medium was changed twice a week. Near cell confluence (80-90\%), cells were washed with PBS and harvested $\left(7 \mathrm{~min}, 37^{\circ} \mathrm{C}\right.$ ) by addition of Accutase (Sigma). hMSC were seeded ( 3000 cells $/ \mathrm{cm}^{2}$ ) on top of fibrous scaffolds and controls $\left(4 \mathrm{~cm}^{2}\right.$ surface area) in 6-well plates (in triplicate).

\subsubsection{Cell staining}

Cells were stained with 4',6-diamidino-2-phenylindole (DAPI) and Phalloidin-TRITC (Sigma) and visualized by inverted fluorescence microscopy. Cells were washed with PBS, fixed with $2 \%(\mathrm{v} / \mathrm{v})$ paraformaldehyde (PFA) for $15 \mathrm{~min}$ and stained with Phalloidin $0.1 \%(\mathrm{v} / \mathrm{v})$ and Saponin 5\% (v/v) in PBS for $1 \mathrm{~h}$, and DAPI 0.15\% (v/v) for 15 min, washed three times with PBS and observed under the microscope.

\subsubsection{Cell viability assay}

At day 5 and 7, cellular metabolic activity was quantified by Alamar Blue cell viability assay (Invitrogen) according with manufacturer's instructions. Culturing cells on a standard tissue culture well and a $4 \mathrm{~cm}^{2}$ glass lamella covered with gelatin (without fibers) were used as positive controls with $4 \mathrm{~cm}^{2}$, and a ultra-low attachment wells (VWR) used as negative control. Flat PHA sheets were used for comparison between scaffold geometries.

\subsubsection{Flow cytometry analysis (FCA)}

hMSC were harvest at day 0 and 7 and flow cytometry analysis (FACScalibur equipment, Becton Dickinson) performed. The cells were stained with human monoclonal antibodies (anti-CD73, antiCD90, anti-CD105) and appropriated anti-IgG isotype control was used.

\subsection{6. hMSC osteo-/-adipogenic differentiation}

hMSC were harvested using Accutase, as described, and cultured in a 24-well culture plate until total cell confluence. The medium was then changed either to (i) DMEM-10\% FBS, $1 \mu \mathrm{M}$ dexamethasone, $50 \mu \mathrm{M}$ 3-isobutyl-1-methyl-xanthine and $200 \mu \mathrm{M}$ indomethacin (Sigma) to induce adipogenic differentiation or (ii) DMEM-10\% FBS, $50 \mu \mathrm{M}$ ascorbate-2-phosphate (Fluka), $10 \mathrm{mM}$ glycerol 2-phosphate disodium salt hydrate (Sigma) to induce osteogenic differentiation. Cells were maintained in DMEM-10\% FBS as control. Medium was changed every 3-4 days. After 2-3 weeks cells were washed with cold PBS, fixed with PFA $2 \%(w / v)$ for 30-15 min, and washed with distilled water. Then cells were either (i) incubated with Oil Red-O solution (Sigma) $(0.3 \%$ (w/v) in isopropanol) for $1 \mathrm{~h}$, washed with distilled water; visualized under optical microscope for red stained lipid vacuoles, typical of mature adipocytes, (ii) submitted to alkaline phosphatase (ALP) activity by incubation with Naphthol AS-MX phosphate solution in fast violet solution (Sigma) $(0.25 \mathrm{mg} / \mathrm{mL}$ ) (1:3 ratio) for $45 \mathrm{~min}$, and for Von Kossa staining by incubation with $2.5 \%(\mathrm{w} / \mathrm{v})$ aqueous silver nitrate solution (Fluka) for $30 \mathrm{~min}$; after each staining, samples are washed with distilled water; visualization with ALP and Von Kossa stainings by optical microscopy allows evaluation of osteogenic commitment and detection of calcium deposits.

\subsection{Scanning electron microscopy (SEM)}

Specimens were coated with a $30 \mathrm{~nm}$ gold layer (Quorum Technologies/Polaron, E5100) prior to SEM analysis using a Hitachi, $\mathrm{S}-2400$ with a $20 \mathrm{kV}$ electron beam and images examined by ImageJ. Cells samples were previously fixed with $2 \%$ PFA, dehydrated by increasing ethanol contents and dried under vacuum before gold coating.

\section{Results}

The terpolymers obtained were characterized in terms of composition, hydrophobicity, stiffness and cytotoxicity and shown to be adequate to support stem cell cultivation.

\subsection{PHA production, composition and characterization}

\subsubsection{PHA production and composition}

Terpolymers of variable monomeric composition were produced in bacterial cultures using different precursor concentrations, DOC and cultivation times [5]. The obtained PHAs were labeled from $A$ to $D$, according to the production batches (Table 1 ). A corresponds to the homopolymer $\mathrm{P}(3 \mathrm{HB}) ; \mathrm{B}, \mathrm{C}$, and $\mathrm{D}$ to polymers with molar percentages, respectively, of $11.4 \%, 28.4 \%$ and $35.6 \%$ in $4 \mathrm{HB}$ and $3.5 \%, 1.7 \%$ and $3.4 \%$ in $3 \mathrm{HV}$ (Fig. 2). PHA cell content, MW and PI are also shown in Fig. 2. In the current study the cultivations were not optimized in terms of volumetric productivity like in some of the previous work [4]. Instead, they were designed to obtain polymers with a range of average monomers composition.

However, it is worth to remark that the highest $\operatorname{Prod}_{\mathrm{vol}}$ was attained in batch $\mathrm{A}$, in the absence of precursors. This batch was the cultivation in which less toxic effects are exerted by the $C$ sources. In batch $\mathrm{B}$, concentrations within 3-10 g/L of GBL were used, together with PA (0-2 g/L), and a terpolymer with an average monomeric molar composition of $85.1,11.4$ and $3.5 \%$ of $3 \mathrm{HB}, 4 \mathrm{HB}$ and $3 \mathrm{HV}$, respectively, was obtained (Fig. 2). The PHA cell content was similar (39.5\%) to that obtained in batch A (38.5\%), but the biomass generated during the growth phase was lower. The later might be ascribed to the inhibiting effect of $1.8 \mathrm{~g} / \mathrm{L}$ of GBL added, at the beginning of the growth phase, for adaptation purposes. This resulted in a lower final PHA concentration of $18.8 \mathrm{~g} / \mathrm{L}$ and a lower $\operatorname{Prod}_{\mathrm{vol}}$ of $0.38 \mathrm{~g} /(\mathrm{Lh})$. The lowest $\operatorname{Prod}_{\mathrm{vol}}(0.21 \mathrm{~g} /(\mathrm{Lh}))$, PHA concentration $(9.3 \mathrm{~g} / \mathrm{L})$ and PHA cell content $(18.1 \%)$ were obtained in batch C, in which DOC was $2 \%$ during the nitrogen starvation stage. GBL concentration was increased to $10-20 \mathrm{~g} / \mathrm{L}$ which led to an increase of the $4 \mathrm{HB}$ molar fraction, resulting in a terpolymer composition of $69.9,28.4$ and $1.7 \%$ of $3 \mathrm{HB}, 4 \mathrm{HB}$ and $3 \mathrm{HV}$, respectively. The terpolymer with the highest $4 \mathrm{HB}$ molar fraction was obtained by extending the cultivation time (batch D). In batch D the PHA content of the cells increased 2-fold due to a 10 -fold increase of the DOC during the PHA production period, which is in agreement with previously published results [5] (Fig. 2). The biopolymers produced presented high PI values. This might be ascribed to their heterogeneous composition since they are, most probably, blends of polymeric chains with different MW and monomer composition [34].

\subsubsection{PHA characterization: mechanical properties and hydrophobicity}

Polymer surface hydrophobicity was assessed by contact angle measurements, and mechanical properties were quantified in terms of elongation at break, stiffness and TSR (Table 2). The contact angle of the terpolymers obtained $\left(73^{\circ}\right.$ and $\left.93^{\circ}\right)$ are not far 

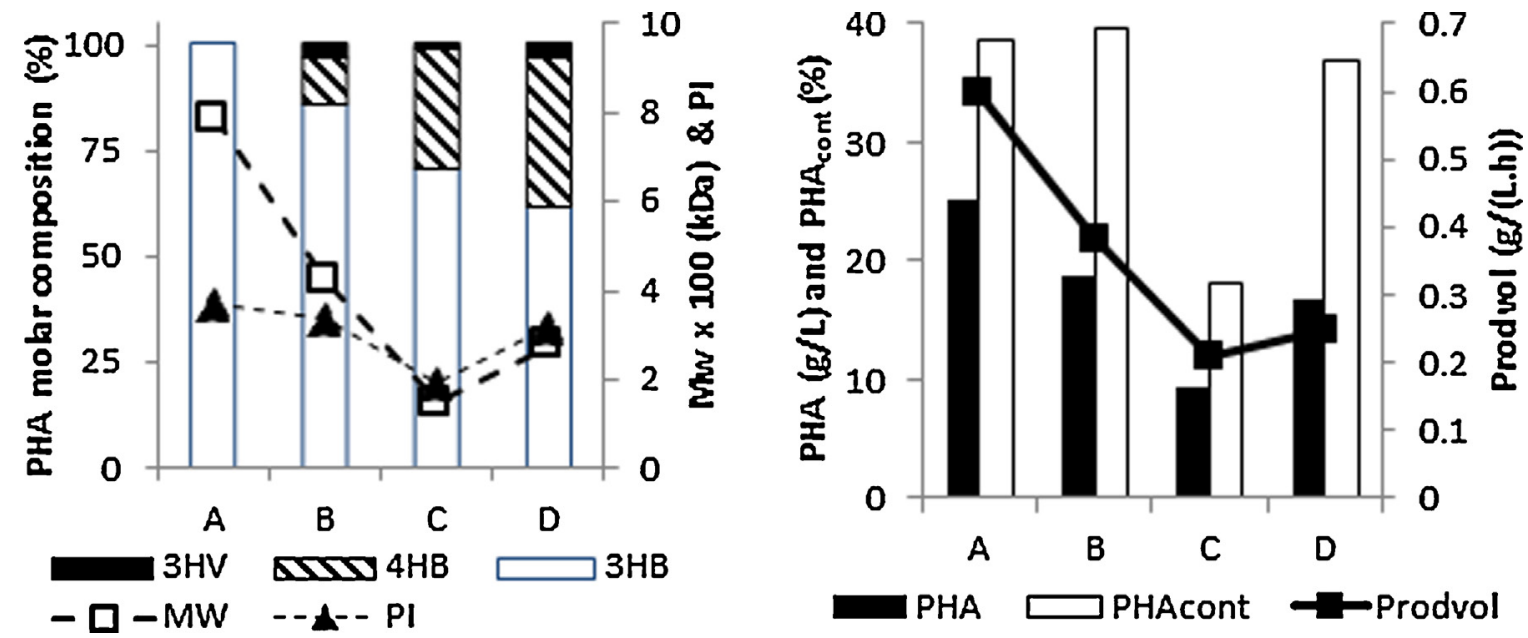

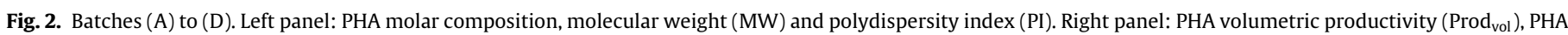
titer and PHA cell content $\left(\mathrm{PHA}_{\text {cont }}\right)$.

Table 2

PHA mechanical properties and contact angles.

\begin{tabular}{|c|c|c|c|c|c|}
\hline Batch & Polymer designation & Contact angle $\left({ }^{\circ}\right)$ & $e(\%)$ & $E(\mathrm{MPa})$ & TSR (MPa) \\
\hline & $\mathrm{P}(3 \mathrm{HB})$ & $67^{\mathrm{a}}$ & $5^{\mathrm{b}}$ & $3500^{\mathrm{b}}$ & $40^{\mathrm{b}}$ \\
\hline B & $\mathrm{P}(3 \mathrm{HB}-11.4 \% 4 \mathrm{HB}-3.5 \% 3 \mathrm{HV})$ & $73.1 \pm 0.5$ & $376 \pm 188$ & $288 \pm 62$ & $11.3 \pm 0.9$ \\
\hline C & $\mathrm{P}(3 \mathrm{HB}-28.4 \% 4 \mathrm{HB}-1.7 \% 3 \mathrm{HV})$ & $92.6 \pm 0.4$ & $76 \pm 11$ & $117 \pm 14$ & $3.9 \pm 0.3$ \\
\hline $\mathrm{D}$ & $\mathrm{P}(3 \mathrm{HB}-35.6 \% 4 \mathrm{HB}-3.4 \% 3 \mathrm{HV})$ & $78.2 \pm 1.5$ & $29 \pm 9$ & $73 \pm 5$ & $2.5 \pm 0.3$ \\
\hline PS tissue culture plates & & $60-70^{c}$ & $2.2^{\mathrm{c}}$ & $3200^{c}$ & - \\
\hline
\end{tabular}

PHAs are labeled B to D, according to the batch where they were synthesized. Elasticity (e), Young's modulus (E) and Tensile strain at Rupture (TSR).

a Volova et al. (2004).

b Lee et al. (1996).

c Suppliers data.

from the value indicated for the polystyrene (PS) tissue culture plates, as well as for that published for $\mathrm{P}(3 \mathrm{HB})$ by Volova [13]. The introduction of the $4 \mathrm{HB}$ moiety seems to slightly increase the polymer hydrophobicity, whereas concomitantly, the two terpolymers with a slightly higher percentage of 3HV show lower contact angles (Table 2). Overall, the contact angles measured for the synthetized PHA fall in the range of values appropriate for cell adhesion as described elsewhere [38], making these polymers potential candidates to support anchorage-dependent cells, such as hMSC. Regarding mechanical properties, the $E$ values found for these PHAs fall in the range between hard cartilage (0.45-0.8 MPa) and bone (11-26 GPa), whereas the PS usually used for cell culture has an $E$ of approximately $3.5 \mathrm{GPa}$. The $E$ values of the terpolymers are within the range $288-73 \mathrm{MPa}$ at $4 \mathrm{HB}$ molar ratios of $11.4-35.6 \%$, and thus their application where desirable cell behavior is induced by less rigid polymers is envisaged. Moreover, $\mathrm{P}(3 \mathrm{HB})$ is a quite hard and resistant though brittle material, with a TSR of $40 \mathrm{MPa}$, while terpolymers B, C and D showed much lower TSRs.

\subsubsection{PHA characterization: cytotoxicity}

The four PHAs obtained in this study were tested for cytotoxicity by indirect and direct contact tests, following ISO10993-5 guidelines. The indirect contact tests (data not shown) show that the lixiviates (i.e. conditioned media) of the four PHAs tested affect the relative metabolic activity of the $\mathrm{L} 929$ to a certain extent; however, relatively to the negative control - fresh culture medium - the values of metabolic activity obtained were above $75 \%$, indicating low cytotoxicity (Fig. 3). Then, direct contact studies were performed in which pieces of the different PHA tested were put on top of a confluent cell layer of L929 cells. These direct tests revealed the absence of inhibitory halos, confirming reduced toxicity (data not shown). Consequently, it was concluded that the four synthetized PHA do not present significant cytotoxic effects and therefore are suitable for cell culture use. This result also indicates that the processing steps used successfully removed most of the solvent.

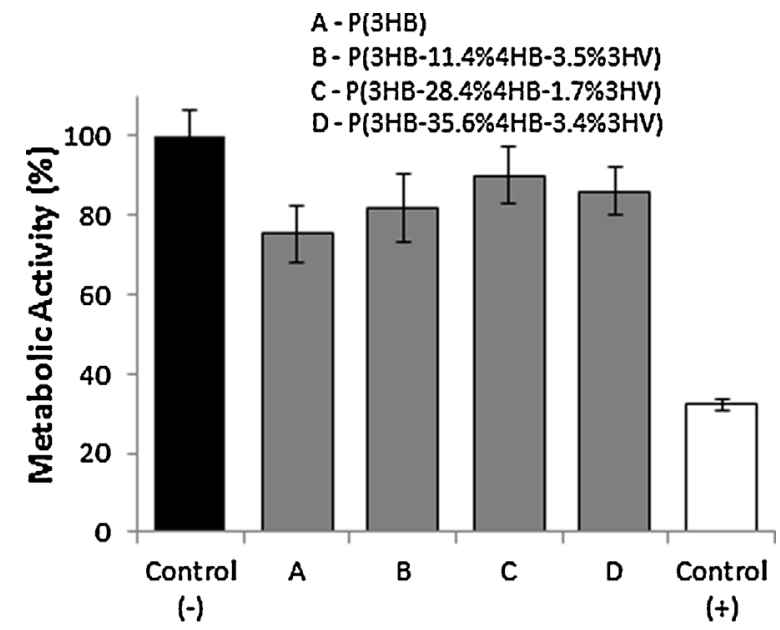

Metabolic activity assessed by MTT test, using $L 929$ mouse fibroblast cell line, $n=4$.

Fig. 3. Indirect cytotoxicity tests. PHAs are labeled as (A) to (D), according to the batch by which they were synthetized. Fresh medium DMEM-10\% FBS and $0.5 \mathrm{v} / \mathrm{v}$ ethanol $96 \%$ were used as negative and positive control, respectively, for the cytotoxicity indirect contact tests. 

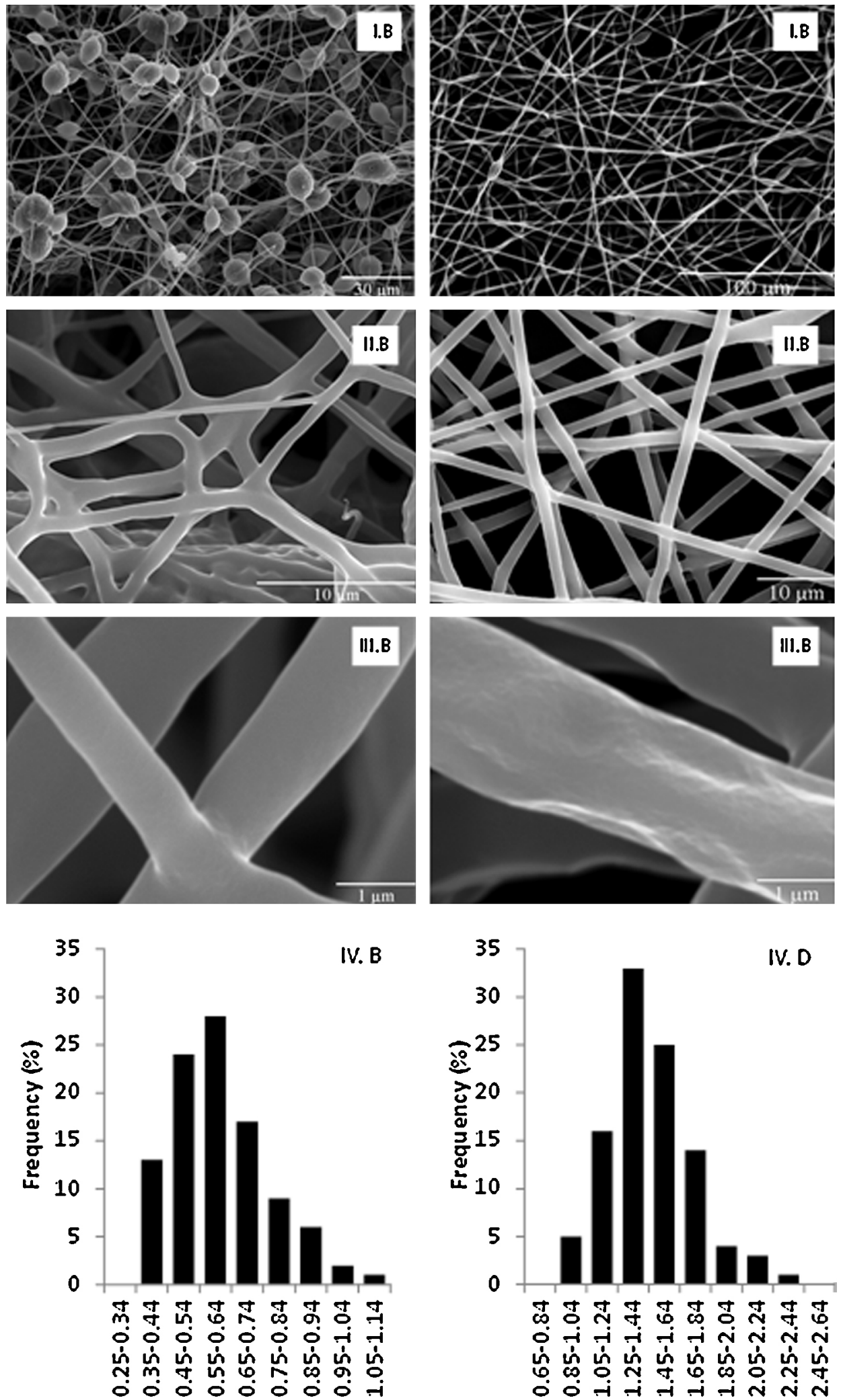

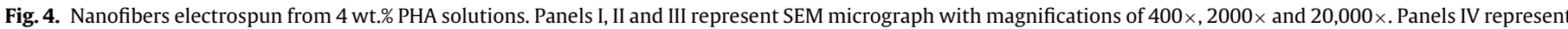
the frequency of fiber diameters for PHA B and D. 


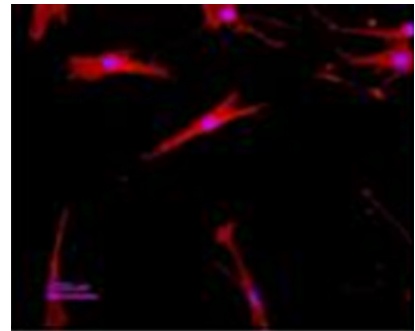

(A) Ctr.PS 200x

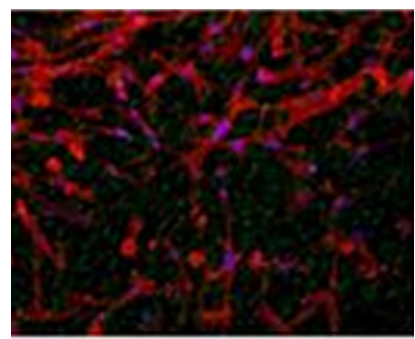

(E) Fibers pol. $8200 x$

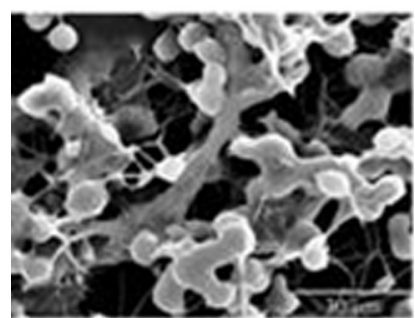

(1)Fibers pd. B $1 \mathrm{Kx}$

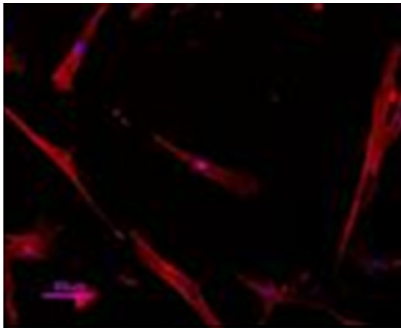

(B) Ctr. Gelatin $200 x$

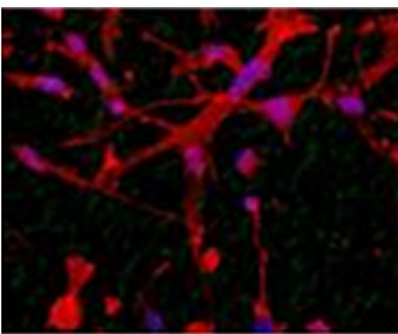

(F) Fibers pol. $8400 x$

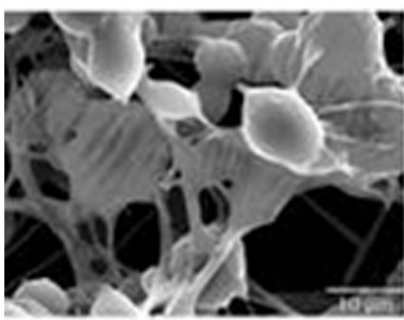

(J) Fibers pd. $82 \mathrm{kx}$

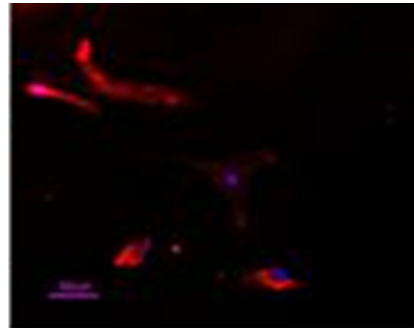

(C) Flat pol. $8200 x$

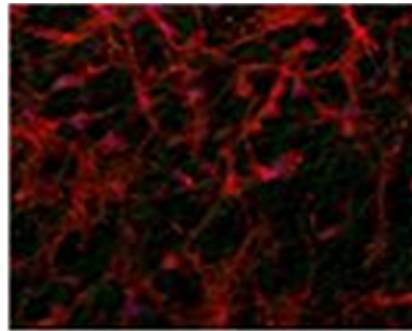

(G)Fibers pol. D 200x

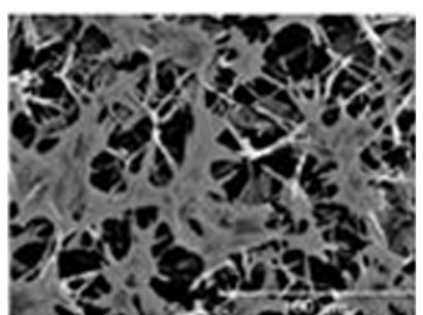

(K) Fibers pol. D 500x

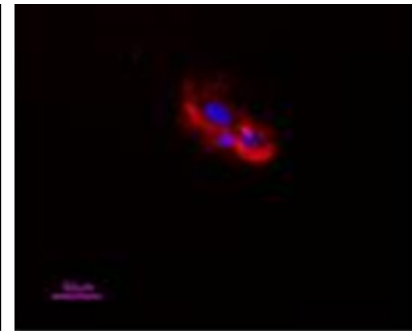

(D) Flat pol D 200x.

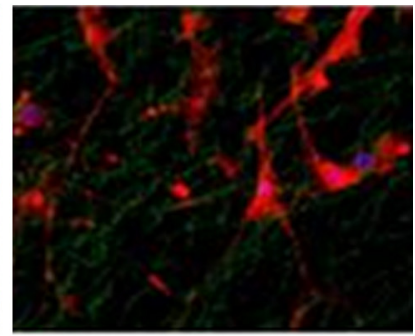

(H)F Fibers pol.D $4000 x$

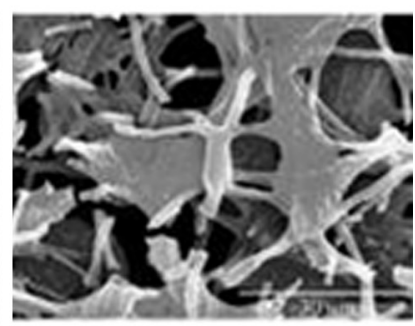

(L)Fibers pol.D 1.5kx

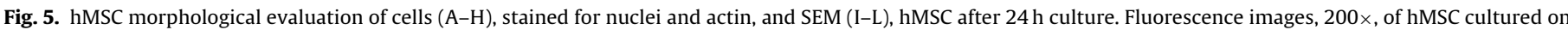

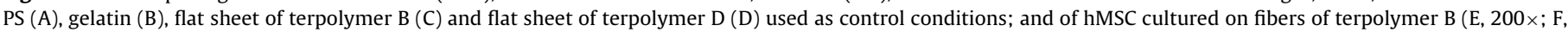

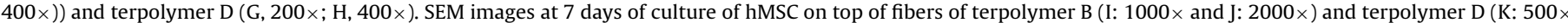
and J: $1500 \times$ ).

\subsection{Fiber mesh architecture and cell adhesion and shape}

\subsubsection{Selection of polymer for electrospinning and fiber mesh} architecture

The PHAs obtained in batch B and D have similar 3HV content, and similar contact angles, but significant differences in $4 \mathrm{HB}$ content and mechanical properties. While the PHA obtained from batch $\mathrm{B}, \mathrm{P}(3 \mathrm{HB}-11.4 \% 4 \mathrm{HB}-3.5 \% 3 \mathrm{HV}), E=288 \mathrm{MPa}$, holds a higher elongation at break and TSR than the other two terpolymers, the PHA obtained in batch D, P(3HB-35.6\%4HB-3.4\%3HV), shows lower stiffness and lower elasticity at break (more brittle). Therefore, polymers from batch $\mathrm{B}$ and $\mathrm{D}$, with substantial differences in stiffness, were selected for further studies, aiming to characterize the fiber meshes produced by electrospinning for their potential to support hMSC culture. In addition flat sheets were also prepared from these two polymers allowing for comparison of material topology. Therefore, to assess hMSC adhesion and proliferation on the PHAs, flat sheets and electrospun fiber meshes were prepared with terpolymers B and D and used as scaffolds for cell culture. Glass slides covered with gelatin and common PS culture plates were used as positive controls.

The SEM images of the resulting fiber meshes and their diameter distribution are illustrated in Fig. 4. Fibers of the stiffer PHA, obtain from batch B, $\mathrm{P}(3 \mathrm{HB}-11.4 \% 4 \mathrm{HB}-3.5 \% 3 \mathrm{HV})$, present a significant amount of beads and their fiber diameter ranges from 350 to $1140 \mathrm{~nm}$, closer to that typically found in natural fibers of the extracellular matrix. Fibers are branched and randomly organized and the mesh exhibits a high porosity and void space interconnectivity, as shown in larger magnifications. The fibrous mesh prepared from the less stiff terpolymer D, presents less branched fibers, with larger fiber diameters, ranging between 850 and $2450 \mathrm{~nm}$. The mean fiber diameter obtained from PHA B and D was around $600 \mathrm{~nm}$ and $1400 \mathrm{~nm}$, respectively.

\subsubsection{Cell adhesion and shape in scaffolds}

To assess hMSC morphology, cells were cultured for $24 \mathrm{~h}$ after which these were stained for their actin filaments and nucleus, using phalloidin (red) and DAPI blue), respectively (Fig. 5A-H), to better examine the cytoskeletal organization. hMSC were able to adhere and spread in all the scaffolds. hMSC presenting a typical elongated, spindle shape were found in both the controls and polymer flat sheets. On both fiber meshes, cells were seen to spread more and along different axes with a branched shape, in contrast with the traditional spindle shape. There was not a specific orientation for the cells on the fiber meshes, as the cytoskeleton was seen to orientate over different axes with different adhesion points, according with the random architecture of the electrospun fibers. Flat sheets of PHA B and D appeared to be less prone to cell adhesion, in particular in the case of terpolymer $D$, which presented very few cells $24 \mathrm{~h}$ after seeding. When compared with the positive controls (i.e. PS plates or gelatin coated surfaces), flat sheets made of polymer B and in particular of polymer D presented a higher number of cells with an unhealthy morphology with a rounder shape 
and not spreading on surface, pointing toward poor adhesion in the first hours of culture.

In order to better examine the cell morphology and their effect on the electrospun fiber meshes, cells were seeded into fiber meshes of both polymers and cultured for 7 days. Fig. 5I-L confirms that a single hMSC spreads and adheres over multiple fibers. On polymer D fiber mesh, hMSC seem to elongate over the larger fibers axis stretching to adhesion points on adjacent fibers (Fig. 5L). On polymer B fibers, it is harder to identify elongation over specific axis. This difference could be accountable to the different sizes of the fibers in both meshes. Polymer D fibers are large enough for a single fiber to allow hMSC growth, while polymer B fiber mesh could have fibers too small to allow it, leading to cell to spread across different fibers without a dominant axis. Still, the beads in polymer B fibers make it impossible to visualize the effect of fiber diameter alone, as the beads provide extra surface area for cells adhesion. Considering the potential MSC spreading along a single fiber, an interesting challenge for PHA electrospinning is to obtain aligned fibers, leading to hMSC orientation in a single direction when regeneration of anisotropic tissue, such as skeletal muscle, is envisaged.

To assess the effects of cell culture on the fiber meshes integrity, samples were taken after 7 days of culture and examined. SEM micrographs (Fig. 5I-J) show cells hanging in long stretches between terpolymer B fibers. This mesh structure made of many beads remains apparently intact and unaltered. Interestingly, in the more uniform terpolymer $\mathrm{D}$ fibrous mesh (with less beads), after 7 days of culture with hMSC, SEM micrographs show broken scaffold fibers (Fig. 5K-L). We hypothesize that the broken fibers observed in Fig. 5K-L are a result of hMSC mechanical action in the fibers, which would be consistent with the mechanical properties of terpolymer D, with a significant lower elongation at break and TSR. Nevertheless, this result can also be an artifact due to sample preparation for SEM. While hydrophobicity of both materials was found to be similar, as shown by the contact angle measurements, terpolymer B is about 4 times harder $(E=288 \mathrm{MPa}$ vs $73 \mathrm{MPa})$ and 13 times more elastic ( $e=379 \%$ vs $29 \%$ ) than terpolymer D. As different material stiffness [39] and geometries [40] have been reported to be capable to specifically tune hMSC into specific cell fates, the high values of Young's modulus of the PHA used (i.e. in the range of 70-300 MPa) are in line with harder tissues, pointing to applications such as bone or tendon regeneration. The ability to obtain different PHAs with a wide range of mechanical properties allows foreseeing potential uses in Regenerative Medicine.

\subsection{Effect of PHA-based scaffolds on hMSC proliferation, immunophenotype and osteo-adipogenic differentiation potential}

\subsubsection{Quantification of hMSC proliferation in scaffolds}

hMSC proliferation was quantified comparatively by measuring the cell population metabolic activity, through the reduction of Resazurin (from Alamar blue kit), which is proportional to the number of cells in culture. Fig. 6 shows values of normalized fluorescence after 7 days of culture for the different samples tested, normalized against measurements of cells cultured on the standard PS tissue plate surface; which presented similar high levels of metabolic activity to cells cultivated in a gelatin coated surfaces. All cell cultures performed on PHA polymers exhibit lower metabolic activities than those on the two controls. The contact tests with L929 mouse fibroblast cells did not indicate cytotoxicity of PHA materials; therefore such lower level of metabolic activity resulted, most probably, from a significantly lower initial adhesion of cells to the PHA as compared to the extent of adhesion both to the optimized standard PS tissue plate surface and to the gelatin surface. Also, during the 7-day period, the cultures performed on the PHA supports show cell proliferation over time (data not shown).

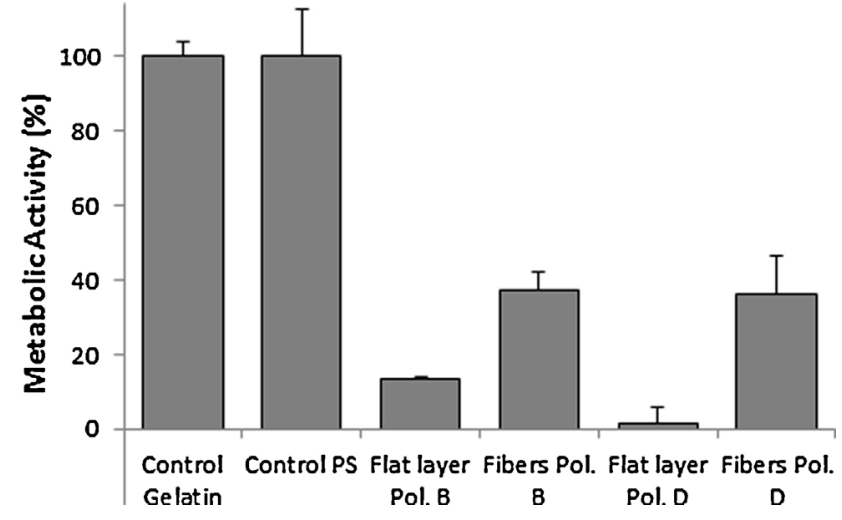

Fig. 6. hMSC metabolic activity (\%) measured by Alamar Blue assay on day 7 of culture.

After 7 days of culture, the number of cells on the flat layer, was negligible for polymer D and low for polymer B. However, significantly higher cell metabolic activities were obtained for hMSC cultured in the fibrous scaffolds, with similar values for both the electrospunned PHAs. The flat sheet made of polymer B was able to support cell culturing, but at about one third of the metabolic activity observed for the fibrous scaffold made such polymer. The higher proliferation estimated for the fibrous meshes can drive from an initial low adhesion of cells to the scaffold than on the flat surface or/and the difficulty to maintain them and promote proliferation on such planar environment. Therefore, for these polymers, the fibrous 3D mesh structure seems to be crucial on supporting cell culture. These results are in agreement with the qualitative information given by Fig. 5C and D vs $\mathrm{G}$ and $\mathrm{H}$ concerning cell morphology. Note that commercial PS tissue plates are optimized for cell adhesion, namely though adjustment of material hydrophobicity, through surface treatment with ionizing irradiation to increase the hydroxyl groups content. The same approach could be applied to the PHA fibrous scaffolds to obtain slightly lower contact angles. Therefore, the metabolic activity attained, at a value of about $36 \%$ of that obtained using optimized PS commercial surfaces is promising, indicating that fibrous scaffolds support an adequate level of cell proliferation.

\subsection{2. hMSC immunophenotype and osteo-adipogenic differentiation potential}

hMSC hold the potential to differentiate into cells of several lineages including cells from the adipose and bone tissues. As hMSC differentiation potential plays an important role on their therapeutic capabilities, it was considered relevant to assess the effect of these materials on differentiation potential of hMSC previously cultivated on PHA-based scaffolds. For that purpose, the cells harvested from the PHA fibrous meshes were characterized by surface markers via flow cytometry analysis and by differentiation assays after culture on the polymer scaffolds for 7 days.

Cell harvesting from the polystyrene tissue cultures, after 7 days of culture, yielded 500 thousand cells with no cells remaining attached upon enzymatic treatment. However, for cultures performed on gelatin and PHA supports, cell harvesting was incomplete, as assessed by microscopic visualization. Upon harvesting, cell viabilities of $96-99 \%$ were obtained for all the samples. Due to limitations on the number of cells retrieved from scaffolds, cell populations harvested from three similar scaffolds cultivated in parallel conditions were pooled together in order to have total cell numbers for a consistent and significant flow cytometry analysis of CD105 expression. The numbers of cells gathered were 125, 100 and 50 thousand for cultures performed on gelatin and fibrous scaffolds made of polymer $\mathrm{B}$ and $\mathrm{D}$, respectively. Considering the low number 


\section{A: CD markers (day 0 )}
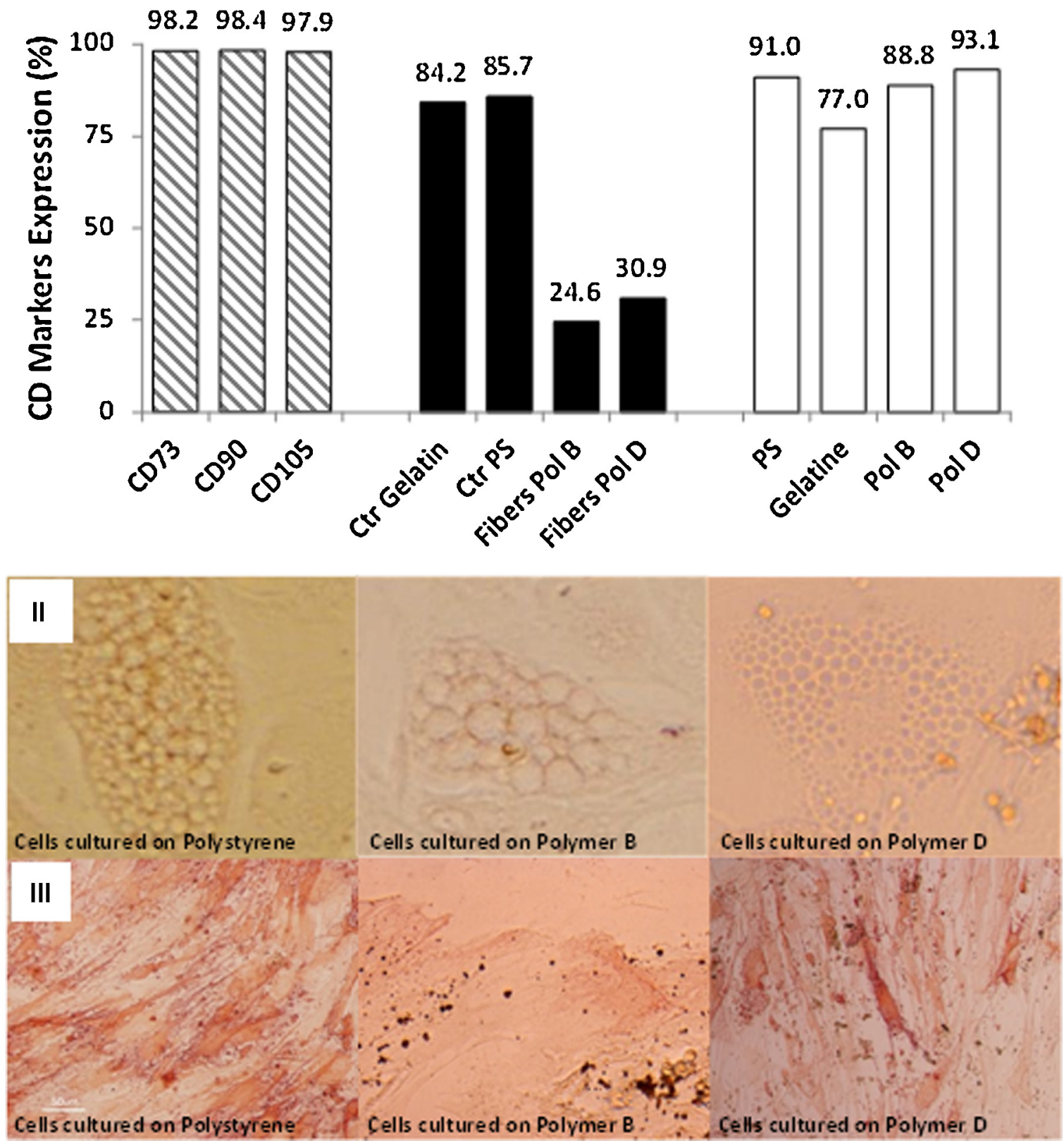

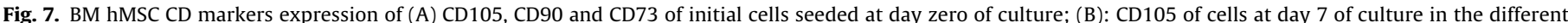

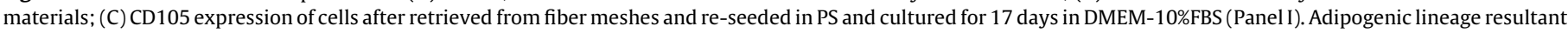

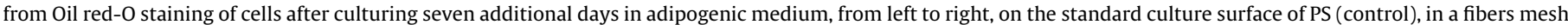

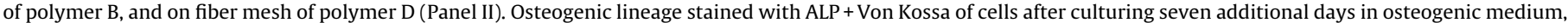
from left to right: on the standard culture surface of PS (control), in a fibers mesh of polymer B, and in a fiber mesh of polymer D.

of cells retrieved from the scaffolds, FCA was performed to evaluate CD105 expression only. In this analysis, we obtained 8500 gated events for cells cultured in gelatin and polystyrene tissue culture plastic, and more than 2200 gated events for fibrous scaffolds at day 7. Concerning the FCA of the seeded cells (day 0 ), more than 10,000 gated events were analyzed for each of the surface antigens typically expressed by human MSC, CD73, CD90 and CD105.

The immunophenotypic characterization of the initial cell population revealed a high expression (above 95\%) for CD73, CD90 and CD105 (Fig. 7A), corresponding to key immunophenotyping defined as minimal criteria to attest MSC identity using flow cytometry [41]. Since a minimal cell number is required to attain statistical significance in flow cytometry analysis and considering the limitations on cell harvesting numbers from the scaffolds by enzymatic treatment, only CD105 expression was analyzed upon cultivation on the different supports. CD105 is a co-receptor for transforming growth factor (TGF) $\beta 1$ and $\beta 3$ highly expressed on hMSC [42]. After 7 days, the cultures supported by PHA fibrous 
scaffolds showed a decrease in CD105 expression, when compared with both controls (Fig. 7B). This result may be a consequence of the enzymatic treatment when harvesting cells, which often damages cell membrane receptors. This is in agreement with previous studies reporting potentially damage of surface cell receptors, e.g. decreasing the number of functional integrins when cells are exposed to prolonged action of proteases and observations from our laboratory where hMSC harvested from chitosan membranes with accutase also revealed a decreased CD105 expression. This loss of CD105 expression by hMSC harvested from PHA-based scaffolds appeared to be reversible since when these cells were put into culture under standard culture conditions (i.e. PS culture wells) for additional 17 days (Fig. 7), the levels of expression of this antigen were restored.

To confirm that hMSC maintain their multipotency, the cells were submitted to differentiation protocols designed to direct cells toward two different lineages: adipogenic and osteogenic. Note that concerning stiffness cues, whereas softer materials promote adipogenesis, osteogenesis is best achieved in hard materials. Therefore, this qualitative assay allows to probe for effects of the material on the MSC commitment. After being harvested from the fibers, cells were cultivated using standard expansion condition. When cells reach confluence, the culture medium was replaced by lineage-specific differentiation medium and cultures maintained for 3 weeks. Adipogenic commitment was confirmed via a high frequency of stained lipid vacuoles using Oil Red-O in the adipogenic induced hMSC. Osteogenic commitment was confirmed by an increase of alkaline phosphatase (ALP) activity, as well the presence of abundant mineralized nodules upon Von Kossa staining in the osteogenic induced hMSC.

\section{Conclusion}

This work demonstrated an integrated strategy for the valorization of a crude glycerol waste stream from a biodiesel plant with production of added-value biocompatible PHA scaffolds, which can be potentially used in Regenerative Medicine and Tissue Engineering. The combination of PHA bioproduction and electrospinning techniques offers a platform for fabrication of biodegradable PHA scaffolds, where mechanical, physical and chemical properties can be tuned and a fibrous 3D structure produced. The four bioproduced polymers were here tested negatively for cytotoxicity and two terpolymers with substantial differences in stiffness were selected to be processed by electrospinning into fibrous meshes with average diameters of $600 \mathrm{~nm}$ and $1400 \mathrm{~nm}$. Comparison of fibrous meshes and flat films made of the same polymer shows that the 3D structure provided by the highly fibrous meshes are crucial on providing focal adhesion points for initial hMSC adherence, organization and proliferation in vitro.

\section{Acknowledgements}

This work was financially supported by the Fundação para a Ciência e Tecnologia (FCT), Portugal through Project PTDC/EQUEQU/114231/2009 and MIT Portugal Program, Bioengineering Systems Focus Area and by the EU Integrated Project BIOPRODUCTION (contract $n^{\circ} 026515-2$ ). The authors thank I. Nogueira, for MicroLabIST SEM analysis and A.P. Serro, IST, for contact angle measurements. J.M.B.T. Cavalheiro, M.C.M.D. de Almeida and F.C. Ferreira gratefully acknowledge the PhD FCT grant SFRH/BD/45266/2008, the Post-doc FCT grant SFRH/BPD/26678/2006, and the Investigador FCT research contract IF/00442/2012, respectively.

\section{References}

[1] S. Marsudi, H. Unno, K. Hori, Appl. Microbiol. Biotechnol. 78 (2008) 955-961.

[2] J. Duque, (Thesis) Design and Analysis of Technological Schemes for Glycerol Conversion to Added Value Products, Universidad Nacional de Colombia, Facultad de Ingeniería y Arquitectura, Departamento de Ingeniería Eléctrica, Electrónica y Computación, 2011.

[3] C. Cardona, J. Posada, M. Montoya, Proceedings of European Congress of Chemical Engineering (ECCE-6), 2007.

[4] J.M.B.T. Cavalheiro, M.C.M.D. de Almeida, C. Grandfils, M.M.R. da Fonseca, Process Biochem. 44 (2009) 509-515.

[5] J.M.B.T. Cavalheiro, R.S. Raposo, M.C.M.D. de Almeida, M.T. Cesário, C. Sevrin, C. Grandfils, M.M.R. da Fonseca, Bioresour. Technol. 111 (2012) 391-397.

[6] S. Yang, K. Leong, Z. Du, C. Chua, Tissue Eng. 7 (2001) 679-689.

[7] B. Demirbag, P.Y. Huri, G.T. Kose, A. Buyuksungur, V. Hasirci, Biotechnol. J. 6 (2011) 1437-1453.

[8] S.Y. Lee, Biotechnol. Bioeng. 49 (1996) 1-14.

[9] E.S. Place, J.H. George, C.K. Williams, M.M. Stevens, Chem. Soc. Rev. 38 (2009) 1139-1151.

[10] A.P. Hollander, S.C. Dickinson, T.J. Sims, P. Brun, R. Cortivo, E. Kon, M. Marcacci, S. Zanasi, A. Borrione, C. De Luca, A. Pavesio, C. Soranzo, G. Abatangelo, Tissue Eng. 12 (7) (2006) 1787-1798.

[11] A. Atala, S.B. Bauer, S. Soker, J.J. Yoo, A.B. Retik, Lancet 367 (9518) (2006) 1241-1246.

[12] S. Chanprateep, K. Buasri, A. Muangwong, P. Utiswannakul, Polym. Degrad. Stab. 95 (2010) 2003-2012.

[13] T.G.Volova, Polyhydroxyalkanoates-Plastic Materials of the 21st Century: Production, Properties, Applications, Nova Science Publishers, Inc., NY, 2004.

[14] S.K. Misra, S.P. Valappil, I. Roy, A.R. Boccaccini, Biomacromolecules 7 (2007) 2249-2258

[15] W. Chen, Y.W. Tong, Acta Biomater. 8 (2012) 540-548.

[16] C. Ye, P. Hu, M.X. Ma, Y. Xiang, R.G. Liu, X.W. Shang, Biomaterials 30 (2009) 4401-4406.

[17] X.Y. Xu, X.T. Li, S.W. Peng, J.F. Xiao, C. Liu, G. Fang, K.C. Chen, G.Q. Chen, Biomaterials 31 (2010) 3967-3975

[18] M. You, G. Peng, J. Li, P. Ma, Z. Wang, W. Shu, S. Peng, G.Q. Chen, Biomaterials 32 (2011) 2305-2313

[19] A. Zonari, S. Novikoff, N.R.P. Electo, N.M. Breyner, D.A. Gomes, A. Martins, N.M Neves, R.L. Reis, A.M. Goes, PLoS ONE 7 (2012) e35422.

[20] X. Wei, Y. Hu, W. Xie, R. Lin, G. Chen, J. Biomed. Mater. Res. 90 (2009) 894-905.

[21] G.-Z. Ji, X. Wei, G.-Q. Chen, J. Biomater. Sci. Polym. Ed. 20 (2009) 325-339.

[22] A.S. Asran, K. Razghandi, N. Aggarwal, G.H. Michler, T. Groth, Biomacromolecules 11 (2010) 3413-3421.

[23] G. Ma, D. Yang, K. Wang, J. Han, S. Ding, G. Song, J. Nie, J. Appl. Polym. Sci. 118 (2010) 3619-3624.

[24] S.K. Misra, T.I. Ansari, S.P. Valappil, D. Mohn, S.E. Philip, W.J. Stark, I. Roy, J.C. Knowles, V. Salih, A.R. Boccaccini, Biomaterials 31 (2005) 2806-2815.

[25] P. Erba, C. Mantovani, D.F. Kalbermatten, G. Pierer, G. Terenghi, P.J. Kingham, J Plast. Reconstr. Aesthet. Surg. 63 (2010) 811-817.

[26] M.F. Pittenger, A.M. Mackay, S.C. Beck, R.K. Jaiswal, R. Douglas, J.D. Mosca, M.A. Moorman, D.W. Simonetti, S. Craig, D.R. Marshak, Science 284 (1999) 143-147.

[27] G. Ferrari, G. Cusella-De Angelis, M. Coletta, E. Paolucci, A. Stornaiuolo, G. Cossu, F. Mavilio, Science 279 (1998) 1528-1530.

[28] T. Toubai, S. Paczesny, Y. Shono, J. Tanaka, K.P. Lowler, C.T. Malter, M. Kasai, M Imamura, Curr. Stem Cell Res. Ther. 4 (2009) 252-259.

[29] D.H. Reneker, I. Chun, Nanotechnology 7 (1996) 216-223.

[30] S.A. Sell, P.S. Wolfe, K. Garg, J.M. McCool, I.A. Rodriguez, G.L. Bowlin, Polymers 2 (2010) 522-553.

[31] C.A. Gregory, J. Ylostalo, D.J. Prockop, Sci STKE 294 (2005) 37.

[32] Y. Wang, R. Gao, P.P. Wang, J. Jian, X.L. Jiang, C. Yan, X. Lin, L. Wu, G.Q. Chen, Q. Wu, Biomaterials 33 (2012) 485-493.

[33] E. Masaeli, M. Morshed, P. Rasekhian, S. Karbasi, K. Karbalaie, F. Karamali, D. Abedi, S. Razavi, A. Jafarian-Dehkordi, M.H. Nasr-Esfahani, H. Baharvand, J. Biomed. Mater. Res A 100 (2012) 1907-1918.

[34] J.M.B.T. Cavalheiro, E. Pollet, H.P. Diogo, M.T. Cesário, L. Avérous, M.C.M.D. de Almeida, M.M.R. da Fonseca, Bioresour. Technol. 147 (2013) 434-441.

[35] A.P. Carapeto, A.P. Serro, B.M.F. Nunes, M.C.L. Martins, S. Todorovice, M.T. Duarte, V. Andréa, R. Colaço, B. Saramago, Surf. Coat. Technol. 204 (2010) $3451-3458$

[36] M. Temtem, L.M. Silva, P.Z. Andrade, F. dos Santos, C.L. da Silva, J.M.S. Cabral, M.M. Abecasis, A. Aguiar-Ricardo, J. Supercrit. Fluids 48 (2008) 269-277.

[37] F. dos Santos, P.Z. Andrade, J.S. Boura, M.M. Abecasis, J.M.S. Cabral, J. Cell Physiol. 223 (2010) 27-35

[38] A. Joy, D.M. Cohen, A. Luk, E. Anim-Danso, C. Chen, J. Kohn, Langmuir 27 (2011) 1891-1899.

[39] A.J. Engler, S. Sen, H.L. Sweeney, D.E. Discher, Cell 126 (2006) 677-689.

[40] Y. Sun, C.S. Chen, J. Fu, Annu. Rev. Biophys. 41 (2012) 519-542.

[41] M. Dominici, K. Le Blanc, I. Mueller, I. Slaper-Cortenbach, F. Marini, D. Krause R. Deans, A. Keating, Dj. Prockop, E. Horwitz, Cytotherapy 8 (2006) 315-317.

[42] H.J. Jin, S.K. Park, W. Oh, Y.S. Yang, S.W. Kim, S.J. Choi, Biochem. Biophys. Res. Commun. 381 (2009) 676-681. 